\title{
Improving cosmological parameter estimation with the future gravitational-wave standard siren observation from the Einstein Telescope
}

\author{
Xuan-Neng Zhang, ${ }^{1}$ Ling-Feng Wang, ${ }^{1}$ Jing-Fei Zhang, ${ }^{1}$ and Xin Zhang*1,2,3,4, ${ }^{\dagger}$ \\ ${ }^{1}$ Department of Physics, College of Sciences, Northeastern University, Shenyang 110819, China \\ ${ }^{2}$ Ministry of Education Key Laboratory of Data Analytics and Optimization for Smart Industry, \\ Northeastern University, Shenyang 110819, China \\ ${ }^{3}$ Center for High Energy Physics, Peking University, Beijing 100080, China \\ ${ }^{4}$ Center for Gravitation and Cosmology, Yangzhou University, Yangzhou 225009, China
}

\begin{abstract}
Detection of gravitational waves produced by merger of binary compact objects could provide an independent way for measuring the luminosity distance to the gravitational-wave burst source, indicating that gravitational-wave observation, combined with observation of electromagnetic counterparts, can provide "standard sirens" for investigating the expansion history of the universe in cosmology. In this work, we wish to investigate how the future gravitational-wave standard siren observations would break the parameter degeneracies existing in the conventional optical observations and how they help improve the parameter estimation in cosmology. We take the third-generation ground-based gravitational-wave detector, the Einstein Telescope, as an example to make an analysis. By simulating 1000 events data in the redshift range between 0 and 5 based on the ten-year observation of the Einstein Telescope, we find that the gravitational-wave data could largely break the degeneracy between the matter density and the Hubble constant, thus significantly improving the cosmological constraints. We further show that the constraint on the equation-of-state parameter of dark energy could also be significantly improved by including the gravitational-wave data in the cosmological fit.
\end{abstract}

The observations from the Planck satellite mission strongly favor a 6-parameter base $\Lambda$ cold dark matter $(\Lambda \mathrm{CDM})$ cosmology [1]. That is to say, in the current stage, one can use only 6 parameters to reproduce the evolution of the universe, including the expansion history and the large-scale structure formation, based on the $\Lambda$ CDM model, which is in good agreement with the current various cosmological observations. However, it is hard to believe that the eventual model of cosmology indeed consists of only 6 parameters. Actually, one believes that the current status is due to the fact that the current observations are not precise enough to tightly constrain other possible parameters beyond the base $\Lambda \mathrm{CDM}$ model, and in the future the base model must be extended in several aspects with the help of future highly accurate observational data.

In fact, it seems that some cracks appear in the base $\Lambda \mathrm{CDM}$ cosmology, which is hinted by the fact that some tensions exist between different astrophysical observations based on the base $\Lambda \mathrm{CDM}$ model, e.g., the wellknown issues concerning $H_{0}$ and $\sigma_{8} \Omega_{\mathrm{m}}^{\alpha}$ (with $\alpha$ taken to be $0.3-0.5)$ measurements [1-3]. A possible way to solve the tensions is to consider some extensions to the base $\Lambda \mathrm{CDM}$ model, but this leads to the fact that the extra parameters are rather difficult to be tightly constrained by the current observational data, in particular, the parameter degeneracies might be strong for these extra parameters. All these facts actually are making requests for the current cosmology: (i) cosmological probes should

\footnotetext{
${ }^{*}$ Corresponding author

$\dagger^{\dagger}$ Electronic address: zhangxin@mail.neu.edu.cn
}

be further developed; and (ii) cosmological model should also be further extended.

Currently, the major cosmological probes mainly include: cosmic microwave background anisotropies (CMB), type Ia supernovae (SN), baryon acoustic oscillations (BAO), direct determination of the Hubble constant, weak gravitational lensing, clusters of galaxies, and redshift-space distortions. The combinations of these cosmological data have provided precise measurements for some cosmological parameters, but for several important extra parameters, e.g., the equation-of-state parameter of dark energy $w(z)$ (with $z$ being redshift), the total mass of neutrinos $\sum m_{\nu}$, the effective number of relativistic species $N_{\text {eff }}$, and so forth, the current observations still cannot provide tight constraints [1]. The future major dark energy experiments (e.g., DESI [4], LSST [5], Euclid [6], WFIRST [7], etc.) will definitely play a crucial role in determining these parameters, but all these experiments are optical (or near-infrared imaging or spectroscopy) observations, which implies that any new observational means would be helpful in avoiding the systematic errors in these optical observations. The promising new cosmological probes mainly include the radio observations (i.e., $21 \mathrm{~cm}$ neutral hydrogen survey) and the gravitational-wave observations.

It is well known from Schutz's work in the mid-1980s [8] that the gravitational waves carry information of cosmic distance of the source. Actually, from the observation of the waveform of gravitational waves released by binary compact objects merger, one can independently measure the luminosity distance to the source of gravitationalwave burst. Furthermore, if the redshift of the source can also be observed by identifying the electromagnetic (EM) counterpart of the source (like the cases of the merger of 
two neutron stars and the merger of a black hole and a neutron star), then one can establish a true distanceredshift relation based on the observation of large amount gravitational-wave events from which the expansion history of the universe can be inferred [9-17]. Compared with the observation of type Ia SN that can also measure the luminosity distance in some sense, the gravitationalwave (GW) observation as a cosmological probe has the following advantages: (i) The SN observation actually cannot measure the absolute luminosity distance, but can only measure the ratio of luminosity distances at different redshifts, due to the fact that the intrinsic luminosity of type Ia SN is not precisely known to us. But the GW observation, definitely, can provide measurement for the absolute luminosity distance to the source. Note also that the measurement of luminosity distance by GW observation is independent of a cosmic distance ladder. (ii) The SN observation can only provide measurements for events with redshifts less than about 1.4, but the GW observation can provide measurements for events with much higher redshifts. These advantages ensure that the future GW observations could play a significant role in breaking the parameter degeneracies and help improve the cosmological parameter estimation.

It should be mentioned here that the detection of the event GW170817 [18], a strong signal from the merger of a binary neutron-star system, by the Advanced LIGO and Virgo detectors demonstrated that the era of multimessenger astronomy has begun. The identification of NGC 4993 as the host galaxy of GW170817 enables us to perform a standard siren measurement of the Hubble constant. Such an independent measurement of the Hubble constant gives the result of $H_{0}=70.0_{-8.0}^{+12.0} \mathrm{~km} \mathrm{~s}^{-1}$ $\mathrm{Mpc}^{-1}[19]$, which is broadly consistent with the existing measurements. This first GW-EM multi-messenger event demonstrates that GW standard siren observations have potential for cosmological inference.

Some forecast studies on cosmology by using the future GW observations (with short-hard $\gamma$-ray bursts or other EM counterparts, as standard sirens) have been performed in the literature (see, e.g., Refs. [20-29]). For example, in Ref. [20], it is shown that the observation from a network of advanced LIGO detectors can constrain the Hubble constant to a 5\% accuracy. In Ref. [21], it is demonstrated that the observation of $1000 \mathrm{GW}$ events from the next-generation ground-based GW detector, the Einstein Telescope (ET), is possible to constrain the cosmological parameters up to $\sigma(h) \sim 5 \times 10^{-3}$ and $\sigma\left(\Omega_{\mathrm{m}}\right) \sim 0.02$ using a Fisher information matrix approach; see also Ref. [23] in which similar results are found using a Markov-chain Monte Carlo (MCMC) method. In Ref. [23], it is found that using the Gaussian Process method the equation-of-state parameter of dark energy can be constrained to be $\sigma[w(z)] \sim 0.03$ in the low redshift region with the future GW observations. Also, in Ref. [29], it is shown that with the help of $1000 \mathrm{GW}$ events observed from the ET the constraints on the neutrino mass can be improved by about 10\%. Furthermore, based on the space-based detector LISA, the expansion of the universe and the interacting dark energy have also been investigated in Refs. [24, 25].

In this work, we wish to investigate how the future GW standard siren observation would break the cosmological parameter degeneracies (existing in the conventional observations) and thus help improve the parameter estimation for cosmology. We will take the third-generation ground-based detector ET as an example to make an analysis. In this analysis, to be in accordance with the previous studies $[21,23,29]$, we will simulate $1000 \mathrm{GW}$ events data in the redshift range of $z \in[0,5]$ based on the ET's ten-year observation. ${ }^{1}$ The GW data simulation method used in this paper is in exact accordance with the prescription given in Refs. [23, 29], and thus we do not repeat it here; we refer the reader to Refs. [23, 29] for details. ${ }^{2}$ There are some parameter degeneracies in cosmological models constrained by the current conventional observations, such as $\mathrm{CMB}, \mathrm{BAO}$, and SN. Using the GW data to make parameter estimation, there will also be some degeneracies, but in this case the orientation of the degeneracy in some parameter plane would be different from the above case. Thus, the GW standard siren observation will play an important role in improving the parameter estimation because it could break parameter degeneracies in the conventional observations. In this paper, we will take the $\Lambda$ CDM model and the $w \mathrm{CDM}$ model [where the equation-of-state parameter (EoS) of dark energy $w$ is taken to be a constant] as examples to see how this happens.

First, we will use the current observations of CMB, $\mathrm{BAO}$, and SN to constrain the two cosmological models, and see how the parameters degenerate with each other, leading to the result that they cannot be well constrained. Then, we will use the simulated GW data from the ET to constrain the models, and we will observe different de-

\footnotetext{
${ }^{1}$ Here we briefly explain why we consider to simulate $1000 \mathrm{GW}$ events data (with EM counterparts). According to the Conceptual Design Study of the Einstein Telescope [30] (see Table 2 on Page 31$)$, the event rate $\left(\mathrm{yr}^{-1}\right)$ in ET is $\mathcal{O}\left(10^{3}-10^{7}\right)$ for coalescences of binary neutron stars (BNS) [and also for neutron star-black hole (NS-BH)]. Following Ref. [21], we here take the event rate in ET to be $10^{5}$ per year for BNS and NS-BH. Considering that short gamma-ray bursts (SGRBs) generated by coalescences of BNS or NS-BH are believed to be beamed with small beaming angle, only a small fraction of the total events are expected to be observed as SGRBs. The fraction for the "useful" events (i.e., the GW events accompanied with the observation of SGRB) is assumed to be $10^{-3}$ [23]. Thus, the number of simulated GW standard siren data considered in this work is $10^{5} \mathrm{yr}^{-1} \times 10^{-3} \times 10 \mathrm{yr}=10^{3}$. See also, e.g., Refs. [21, 23, 29].

2 Note here that in the simulation we mainly consider the coalescence events of BNS and also consider a small number of NS-BH coalescence events. According to the prediction of the Advanced LIGO-Virgo network, we take the ratio of the events numbers of NS-BH and BNS to be 0.03. For the mass distributions of NS and $\mathrm{BH}$, we randomly sample the mass of NS in the interval of $[1,2] M_{\odot}$ and the mass of $\mathrm{BH}$ in the interval of $[3,10] M_{\odot}$, where $M_{\odot}$ is the solar mass, in accordance with Refs. [23, 29].
} 

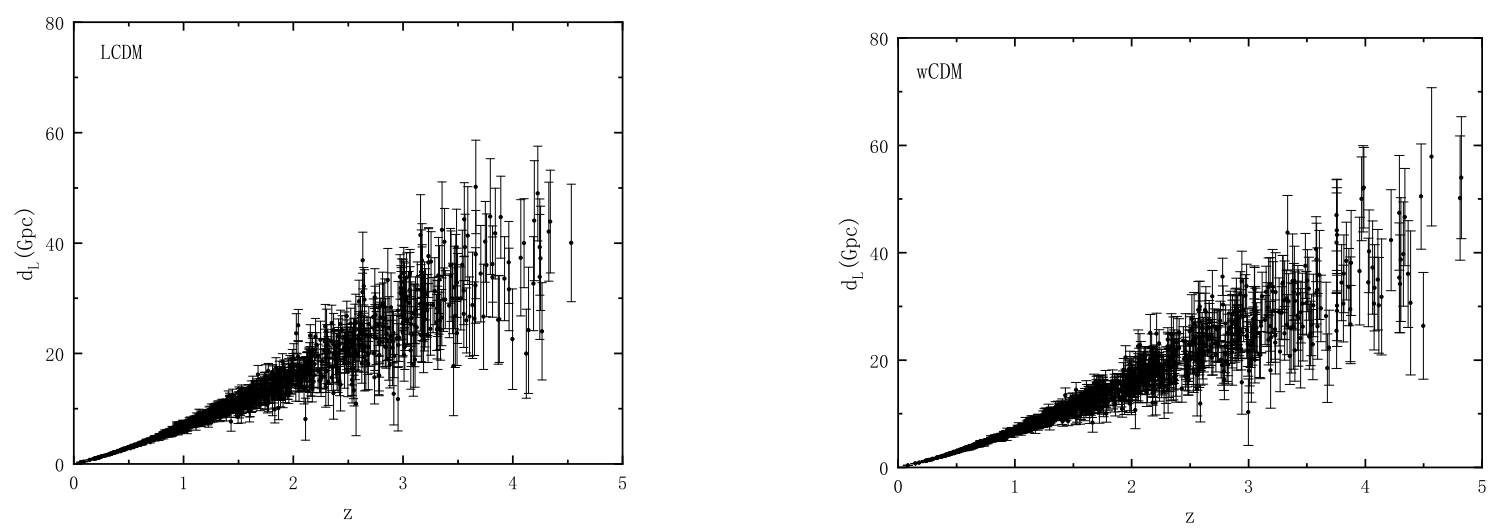

FIG. 1: The luminosity distance data points from simulated 1000 gravitational-wave events in the redshift range of $z \in[0,5]$ based on the ten-year observation of the Einstein Telescope. The fiducial models are chosen as the best-fitted $\Lambda$ CDM (left) and $w \mathrm{CDM}$ (right) models, respectively, constrained by the current $\mathrm{CMB}+\mathrm{BAO}+\mathrm{SN}$ data.

TABLE I: Fitting results for the $\Lambda \mathrm{CDM}$ model and the $w \mathrm{CDM}$ model using CBS, GW, and CBS+GW. Here, CBS stands for $\mathrm{CMB}+\mathrm{BAO}+\mathrm{SN}$.

\begin{tabular}{|c|c|c|c|c|c|c|}
\hline Model & \multicolumn{3}{|c|}{$\overline{\Lambda C D M}$} & \multicolumn{3}{|c|}{$w \mathrm{CDM}$} \\
\hline Data & CBS & GW & CBS+GW & CBS & GW & $\mathrm{CBS}+\mathrm{GW}$ \\
\hline$\Omega_{\mathrm{b}} h^{2}$ & $0.02233 \pm 0.00014$ & $0.050_{-0.045}^{+0.050}$ & $0.02229 \pm 0.00010$ & $0.02230 \pm 0.00015$ & $0.051_{-0.046}^{+0.049}$ & $0.02231 \pm 0.00013$ \\
\hline$\Omega_{\mathrm{c}} h^{2}$ & $0.11853 \pm 0.00101$ & $0.091_{-0.025}^{+0.038}$ & $0.11898_{-0.00051}^{+0.00050}$ & $0.11893_{-0.00125}^{+0.00124}$ & $0.081_{-0.029}^{+0.040}$ & $0.11872_{-0.00104}^{+0.00105}$ \\
\hline $100 \theta_{\mathrm{MC}}$ & $1.04092 \pm 0.00030$ & $0.96_{-0.11}^{+0.12}$ & $1.04086_{-0.00026}^{+0.00027}$ & $1.04087 \pm 0.00030$ & $0.95 \pm 0.11$ & $1.04088 \pm 0.00028$ \\
\hline$\tau$ & $0.086 \pm 0.016$ & - & $0.083 \pm 0.016$ & $0.084 \pm 0.017$ & - & $0.084 \pm 0.017$ \\
\hline$n_{\mathrm{s}}$ & $0.9679_{-0.0040}^{+0.0039}$ & - & $0.9666_{-0.0033}^{+0.0032}$ & $0.9668 \pm 0.0044$ & - & $0.9673 \pm 0.0040$ \\
\hline $\ln \left(10^{10} A_{\mathrm{s}}\right)$ & $3.103 \pm 0.032$ & - & $3.098 \pm 0.032$ & $3.101 \pm 0.033$ & - & $3.101 \pm 0.033$ \\
\hline$\Omega_{\mathrm{m}}$ & $0.3075_{-0.0061}^{+0.0060}$ & $0.3119_{-0.0073}^{+0.0071}$ & $0.3103 \pm 0.0024$ & $0.3039_{-0.0093}^{+0.0094}$ & $0.2890 \pm 0.0150$ & $0.3061 \pm 0.0023$ \\
\hline$H_{0}[\mathrm{~km} / \mathrm{s} / \mathrm{Mpc}]$ & $67.84 \pm 0.46$ & $67.57 \pm 0.22$ & $67.62 \pm 0.16$ & $68.3_{-1.1}^{+1.0}$ & $67.79_{-0.33}^{+0.32}$ & $68.04_{-0.24}^{+0.23}$ \\
\hline$w$ & - & - & - & $-1.023_{-0.041}^{+0.042}$ & $-0.943_{-0.054}^{+0.062}$ & $-1.010 \pm 0.020$ \\
\hline
\end{tabular}
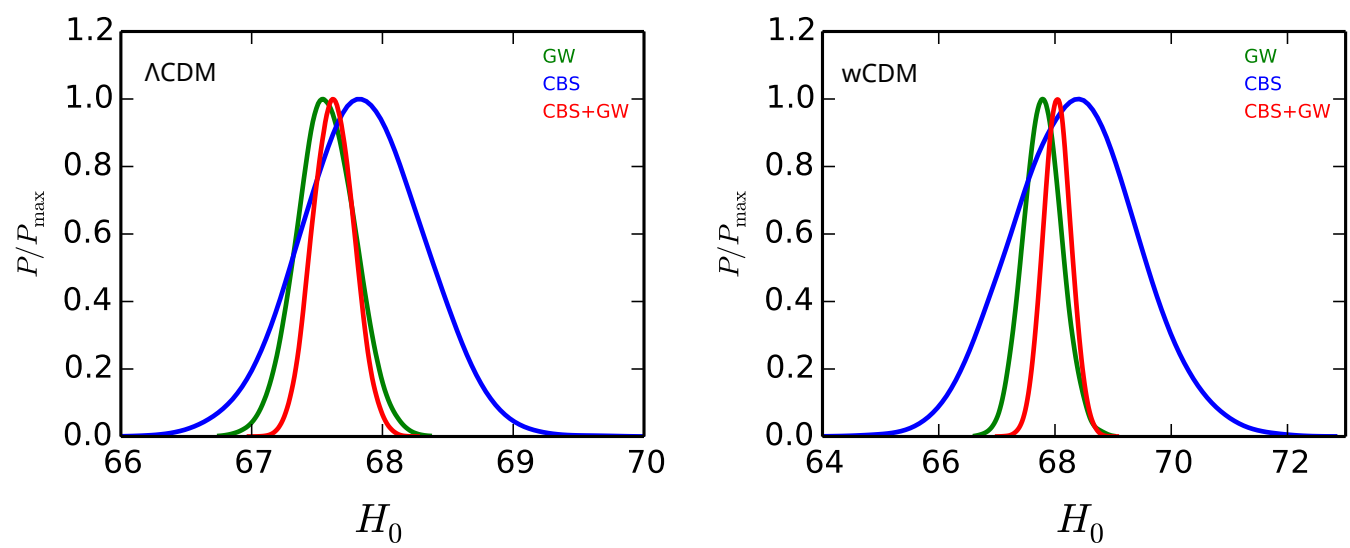

FIG. 2: One-dimensional posterior distributions of $H_{0}$ in the $\Lambda$ CDM model (left) and the $w$ CDM model (right) using the CBS, $\mathrm{GW}$, and $\mathrm{CBS}+\mathrm{GW}$ data combinations. Here, CBS stands for $\mathrm{CMB}+\mathrm{BAO}+\mathrm{SN}$.

generacy cases, which leads to the fact that the previous degeneracies are broken when the GW data are included in the data combination.

We now briefly describe the current observations used in this paper. For CMB data, we use the Planck temperature and polarization power spectra (Planck TT,TE,EE+lowP) [1]. For BAO data, we use the measurements from 6dFGS $\left(z_{\text {eff }}=0.106\right)$ [31], SDSS-MGS 

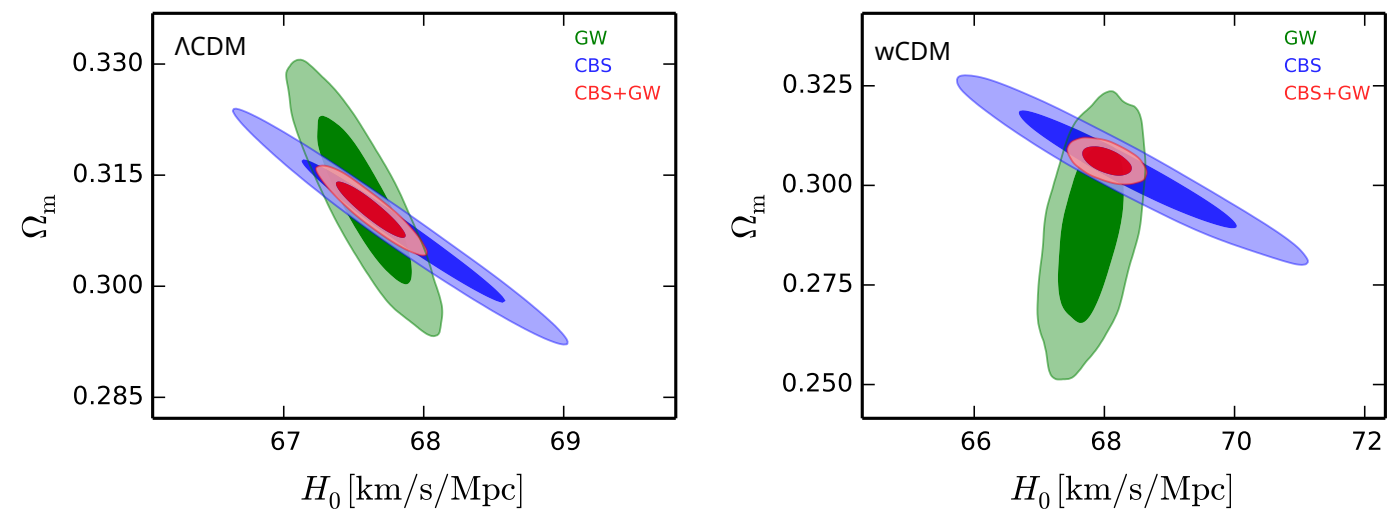

FIG. 3: Constraints $(68.3 \%$ and $95.4 \% \mathrm{CL})$ on the $\Lambda$ CDM model (left) and the $w$ CDM model $($ right $)$ in the $H_{0}-\Omega_{\mathrm{m}}$ plane using the $\mathrm{CBS}, \mathrm{GW}$, and $\mathrm{CBS}+\mathrm{GW}$ data combinations. Here, CBS stands for $\mathrm{CMB}+\mathrm{BAO}+\mathrm{SN}$.

TABLE II: Constraint errors and accuracies for parameters of $\Lambda \mathrm{CDM}$ and $w \mathrm{CDM}$ using $\mathrm{CBS}, \mathrm{GW}$, and CBS+GW. Here, CBS stands for $\mathrm{CMB}+\mathrm{BAO}+\mathrm{SN}$.

\begin{tabular}{cccccccc}
\hline \hline Model & \multicolumn{4}{c}{$\Lambda$ CDM } & & \multicolumn{3}{c}{$w$ CDM } \\
\cline { 7 - 8 } \cline { 6 - 7 } Data & CBS & GW & CBS+GW & CBS & GW & CBS+GW \\
\hline$\sigma\left(\Omega_{\mathrm{m}}\right)$ & 0.0060 & 0.0072 & 0.0024 & & 0.0094 & 0.0150 & 0.0023 \\
$\sigma(h)$ & 0.0046 & 0.0022 & 0.0016 & & 0.0105 & 0.0033 & 0.0024 \\
$\sigma(w)$ & - & - & - & 0.042 & 0.058 & 0.020 \\
$\varepsilon\left(\Omega_{\mathrm{m}}\right)$ & 0.0197 & 0.0231 & 0.0077 & 0.0308 & 0.0520 & 0.0075 \\
$\varepsilon(h)$ & 0.0068 & 0.0033 & 0.0024 & 0.0153 & 0.0048 & 0.0035 \\
$\varepsilon(w)$ & - & - & - & 0.041 & 0.062 & 0.020 \\
\hline \hline
\end{tabular}

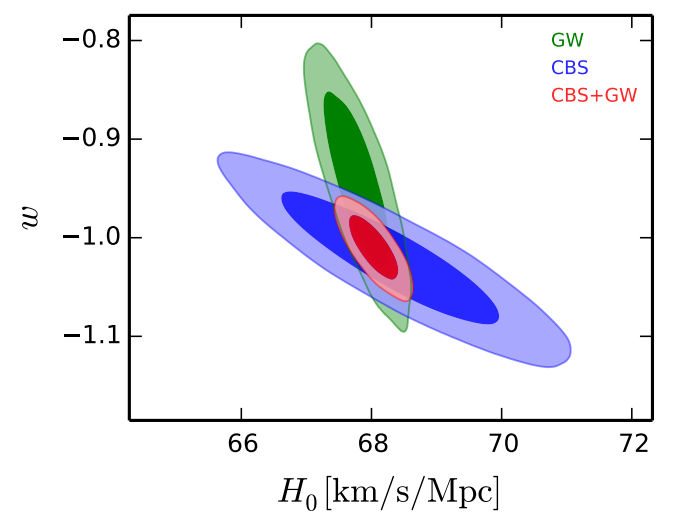

FIG. 4: Constraints $(68.3 \%$ and $95.4 \% \mathrm{CL})$ on the $w \mathrm{CDM}$ model in the $H_{0}-w$ plane using the CBS, GW, and CBS+GW data combinations. Here, CBS stands for CMB+BAO+SN.

$\left(z_{\text {eff }}=0.15\right)[32]$, and BOSS LOWZ $\left(z_{\text {eff }}=0.32\right)$ and CMASS $\left(z_{\text {eff }}=0.57\right)$ [33]. For SN data, we use the JLA compilation [34]. We use the data combination of $\mathrm{CMB}+\mathrm{BAO}+\mathrm{SN}$ to constrain the $\Lambda \mathrm{CDM}$ and $w \mathrm{CDM}$ models by employing the MCMC package CosmoMC [35], and then take the best-fitted models as the fiducial models to generate the simulated GW data. In Fig. 1, we show the simulated GW data consisting of $d_{L}^{\text {mea }}$ and $\sigma_{d_{L}}$ in the redshift range of $z \in[0,5]$ for the two cases in which the $\Lambda \mathrm{CDM}$ model and the $w \mathrm{CDM}$ model are taken as fiducial models, respectively.

We then use the simulated GW data to constrain the models. The constraints from the data combination of $\mathrm{CMB}+\mathrm{BAO}+\mathrm{SN}+\mathrm{GW}$ will show how the GW data help improve the parameter estimation in the considered two cases. We summarize the fitting results in Tables I and II. In Table I we show the fitting values of cosmological parameters, and in Table II we show the constraint errors and constraint accuracies for the concerned parameters (i.e., $\Omega_{\mathrm{m}}, H_{0}$, and $w$ ). Here, the error $\sigma$ is taken to be the average of $\sigma_{+}$and $\sigma_{-}$, and $\varepsilon(\xi)$ for a parameter $\xi$ is defined as $\varepsilon(\xi)=\sigma(\xi) / \xi$.

In Fig. 2, we show the one-dimensional posterior distributions of $H_{0}$ in the $\Lambda \mathrm{CDM}$ model (left) and the $w \mathrm{CDM}$ model (right) using the $\mathrm{CMB}+\mathrm{BAO}+\mathrm{SN}, \mathrm{GW}$, and $\mathrm{CMB}+\mathrm{BAO}+\mathrm{SN}+\mathrm{GW}$ data combinations. Note that, for convenience, hereafter we use the abbreviation "CBS" to denote the combination $\mathrm{CMB}+\mathrm{BAO}+\mathrm{SN}$. Here we can clearly see that the GW observation solely can tightly constrain the Hubble constant. In the case of $\Lambda$ CDM, we have $\sigma(h)=4.6 \times 10^{-3}$ and $\varepsilon(h)=0.68 \%$ from CBS, $\sigma(h)=2.2 \times 10^{-3}$ and $\varepsilon(h)=0.33 \%$ from $\mathrm{GW}$, and $\sigma(h)=1.6 \times 10^{-3}$ and $\varepsilon(h)=0.24 \%$ from $\mathrm{CBS}+\mathrm{GW}$. We find that the accuracy of $H_{0}$ is improved from $0.68 \%$ to $0.24 \%$ in the $\Lambda$ CDM case when the GW data are included in the fit. In the case of $w \mathrm{CDM}$, we have $\sigma(h)=1.05 \times 10^{-2}$ and $\varepsilon(h)=1.53 \%$ from CBS, $\sigma(h)=3.3 \times 10^{-3}$ and $\varepsilon(h)=0.48 \%$ from GW, and $\sigma(h)=2.4 \times 10^{-3}$ and $\varepsilon(h)=0.35 \%$ from CBS+GW. We find that the accuracy of $H_{0}$ is improved from $1.53 \%$ to $0.35 \%$ in the $w \mathrm{CDM}$ case when the GW data are included in the fit. Obviously, it is shown from this analysis that the GW observation is capable of significantly improve the constraint accuracy of $H_{0}$, in particular in dynamical dark energy models.

Figure 3 shows constraints on the $\Lambda \mathrm{CDM}$ model (left) and the $w \mathrm{CDM}$ model (right) in the $H_{0}-\Omega_{\mathrm{m}}$ plane. In this figure, we can clearly see that, from the CBS con- 
straint, in both the $\Lambda \mathrm{CDM}$ and $w \mathrm{CDM}$ models, $\Omega_{\mathrm{m}}$ and $H_{0}$ are in strong anti-correlation. In the case of $\Lambda \mathrm{CDM}$, the GW constraint still gives an anti-correlation for $\Omega_{\mathrm{m}}$ and $H_{0}$, but its degeneracy orientation in the parameter plane is evidently different from the former, resulting in the breaking of the degeneracy. In the case of $w \mathrm{CDM}$, we find that the GW constraint leads to a weak positive correlation for $\Omega_{\mathrm{m}}$ and $H_{0}$, and thus the orthogonality of the two degeneracy orientations results in a complete breaking of the parameter degeneracy. Thus, although CBS and GW give similar constraints on $\Omega_{\mathrm{m}}$, the combination of the two could tremendously improve the constraint on $\Omega_{\mathrm{m}}$. In the $\Lambda \mathrm{CDM}$ case the constraint on $\Omega_{\mathrm{m}}$ is improved from $1.97 \%$ to $0.77 \%$, and in the $w \mathrm{CDM}$ case the constraint on $\Omega_{\mathrm{m}}$ is from $3.08 \%$ to $0.75 \%$, when the GW data are included in the fit.

In Fig. 4, we show the constraints on the $w \mathrm{CDM}$ model in the $H_{0}-w$ plane. From this figure, we can also clearly see that the parameter degeneracy could be largely broken by including the GW observation in the cosmological fit. The CBS data give $\sigma(w)=0.042$ and the $\mathrm{GW}$ data give $\sigma(w)=0.058$, indicating that their constraints on $w$ are similar. But the combination of the two gives $\sigma(w)=0.020$, showing that the error is largely decreased. By considering the GW observation, the constraint accuracy of $w$ is improved from $4.1 \%$ to $2.0 \%$. Therefore, in this analysis, we have shown that the GW observation could help greatly improve the constraint accuracy of $w$.

Finally, let us make some relevant discussions. GW standard sirens have some advantages in studying cosmology. For example, GW standard sirens are selfcalibrating and thus have no dependence on a cosmic distance ladder, which leads to that observation of GW standard sirens can measure true luminosity distances and establish a true distance-redshift relation to study cosmography. Moreover, compared with SN observation, GW observation can measure sources with higher redshifts. Although we know that GW standard sirens have these advantages, we still need to confirm the usefulness of GW standard sirens in precision cosmology. The core aim of this work is to investigate what role the future observation of GW standard sirens can play in the study of precision cosmology.

Compared with previous studies in the literature, the feature of this work is to be with a view to the question of whether the GW observation from ET can break the parameter degeneracies existing in the EM observations. In Ref. [21] it was found by using a Fisher matrix approach that ET (combined with the Planck CMB prior) will be able to constrain $w_{0}$ and $w_{a}$ [for the dynamical dark energy model with parameterization $w(a)=w_{0}+w_{a}(1-a)$ with $a$ being the scale factor of the universe] with the errors $\sigma\left(w_{0}\right)=0.099$ and $\sigma\left(w_{a}\right)=0.302$, respectively, and these results are comparable with the projected errors for the JDEM BAO project and the SNAP SN observations. In Ref. [23] it was found by using a MCMC approach that the Gaussian Process method can help reconstruct the evolution of EoS of dark energy and the future GW observation from ET would give $\sigma[w(z)] \sim 0.03$ in the low redshift region. However, in the present study we wish to focus on the issue of the role of GW observation in precision cosmology. We investigate what the role the future ET GW observation would play in constraining cosmological parameters in combination with EM observations. We only employ the simplest cosmological models, i.e., the $\Lambda \mathrm{CDM}$ model and the $w \mathrm{CDM}$ model, to complete the analysis, in which a MCMC method is used. It is shown in this work that some parameter degeneracies can be successfully broken by using the GW observation. Although the GW observation can be used to precisely determine the Hubble constant $H_{0}$, for other cosmological parameters the GW observation actually cannot provide very tight constraints. For example, the GW observation can only constrain $\Omega_{\mathrm{m}}$ and $w$ (in the $w \mathrm{CDM}$ model) at the accuracies of $5.20 \%$ and $6.2 \%$, respectively, while the current $\mathrm{CMB}+\mathrm{BAO}+\mathrm{SN}$ observation can constrain them at the accuracies of $3.08 \%$ and $4.1 \%$, respectively, actually better than the former. However, owing to the fact that the degeneracy orientations from GW and EM observations in the parameter space are distinctively different from each other, the parameter degeneracies can be well broken, which leads to that the combined data of GW and EM can constrain $\Omega_{\mathrm{m}}$ and $w$ at the accuracies of $0.75 \%$ and $2.0 \%$, respectively. Therefore, the goal of this work is achieved: We have concluded from our analysis that the role of the future ET GW observation in the precision cosmology is to break the parameter degeneracies of the EM observations, and the combination of GW and EM observations can provide much better constraints on cosmological parameters.

Actually, using only a small number of low-redshift GW events produced by BNSs can precisely determine the value of the Hubble constant $H_{0}$ in a modelindependent way. This will definitely resolve the $H_{0}$ tension and judge if the $\Lambda \mathrm{CDM}$ model should be extended. A recent study [28] pointed out that about 50 BNS standard sirens data will be able to fulfill this task. In the present study we have pointed out that in extensions to $\Lambda \mathrm{CDM}$ cosmology the parameter degeneracies (in particular for the extra parameters) can be well broken by the future GW standard sirens observation. It is expected that in the future the combination of GW and EM observations would push the precision cosmology forward by a great step.

In summary, it is shown in this work that the future GW standard siren observation is capable of breaking the parameter degeneracies existing in the conventional optical observations and thus could help improve the parameter estimation for cosmology. We have simulated 1000 GW events data based on the ET's ten-year observation. In order to show how the GW data break the parameter degeneracies, we employ the current $\mathrm{CMB}+\mathrm{BAO}+\mathrm{SN}$ data to make comparison and combination. We take the $\Lambda \mathrm{CDM}$ and $w \mathrm{CDM}$ models as examples to make an analysis. We find that the degeneracy between $\Omega_{\mathrm{m}}$ and $H_{0}$ can be greatly broken by including the GW data, partic- 
ularly for the case of $w$ CDM. Thus, for both $\Omega_{\mathrm{m}}$ and $H_{0}$, the constraint accuracies are tremendously improved by considering the $\mathrm{GW}$ data from ET. Although $w$ is hard to be tightly constrained, the GW observation from ET could help improve its constraint accuracy from $4 \%$ to $2 \%$, according to our analysis. In this work, we only make a preliminary analysis, because we only consider the improvement for the parameter estimation based on the current $\mathrm{CMB}+\mathrm{BAO}+\mathrm{SN}$ data, but not the future optical observations. Moreover, we only consider the simplest dynamical dark energy model, i.e., the $w \mathrm{CDM}$ model, in this work. We will leave a comprehensive analysis to a future work.

\section{Acknowledgments}

We would like to thank Zhou-Jian Cao, Tao Yang, and Wen Zhao for helpful discussions. This work was supported by the National Natural Science Foundation of China (Grants Nos. 11875102, 11835009, 11522540, and 11690021) and the National Program for Support of TopNotch Young Professionals.
[1] N. Aghanim et al. [Planck Collaboration], "Planck 2015 results. XI. CMB power spectra, likelihoods, and robustness of parameters," Astron. Astrophys. 594, A11 (2016) doi:10.1051/0004-6361/201526926 [arXiv:1507.02704 [astro-ph.CO]].

[2] P. A. R. Ade et al. [Planck Collaboration], "Planck 2013 results. XVI. Cosmological parameters," Astron. Astrophys. 571, A16 (2014) doi:10.1051/0004-6361/201321591 [arXiv:1303.5076 [astro-ph.CO]].

[3] M. J. Mortonson, D. H. Weinberg and M. White, "Dark Energy: A Short Review," arXiv:1401.0046 [astroph.CO].

[4] M. Levi et al. [DESI Collaboration], "The DESI Experiment, a whitepaper for Snowmass 2013," arXiv:1308.0847 [astro-ph.CO].

[5] P. A. Abell et al. [LSST Science and LSST Project Collaborations], "LSST Science Book, Version 2.0," arXiv:0912.0201 [astro-ph.IM].

[6] R. Laureijs et al. [EUCLID Collaboration], "Euclid Definition Study Report," arXiv:1110.3193 [astro-ph.CO].

[7] D. Spergel et al., "Wide-Field InfraRed Survey Telescope-Astrophysics Focused Telescope Assets WFIRST-AFTA Final Report," arXiv:1305.5422 [astroph.IM].

[8] B. F. Schutz, "Determining the Hubble Constant from Gravitational Wave Observations," Nature 323, 310 (1986). doi:10.1038/323310a0.

[9] D. E. Holz and S. A. Hughes, "Using gravitationalwave standard sirens," Astrophys. J. 629, 15 (2005) doi:10.1086/431341 [astro-ph/0504616].

[10] N. Dalal, D. E. Holz, S. A. Hughes and B. Jain, "Short GRB and binary black hole standard sirens as a probe of dark energy," Phys. Rev. D 74, 063006 (2006) doi:10.1103/PhysRevD.74.063006 [astro-ph/0601275].

[11] C. L. MacLeod and C. J. Hogan, "Precision of Hubble constant derived using black hole binary absolute distances and statistical redshift information," Phys. Rev. D 77, 043512 (2008) doi:10.1103/PhysRevD.77.043512 [arXiv:0712.0618 [astro-ph]].

[12] B. S. Sathyaprakash, B. F. Schutz and C. Van Den Broeck, "Cosmography with the Einstein Telescope," Class. Quant. Grav. 27, 215006 (2010) doi:10.1088/02649381/27/21/215006 [arXiv:0906.4151 [astro-ph.CO]].

[13] S. R. Taylor, J. R. Gair and I. Mandel, "Hubble without the Hubble: Cosmology using advanced gravitationalwave detectors alone," Phys. Rev. D 85, 023535 (2012)
doi:10.1103/PhysRevD.85.023535 [arXiv:1108.5161] [gr$\mathrm{qc}]]$.

[14] W. Del Pozzo, "Inference of the cosmological parameters from gravitational waves: application to second generation interferometers," Phys. Rev. D 86, 043011 (2012) doi:10.1103/PhysRevD.86.043011 [arXiv:1108.1317 [astro-ph.CO]].

[15] C. Messenger, K. Takami, S. Gossan, L. Rezzolla, and B. S. Sathyaprakash, "Source Redshifts from Gravitational-Wave Observations of Binary Neutron Star Mergers," Phys. Rev. X 4, 041004 (2014) doi:10.1103/PhysRevX.4.041004.

[16] W. Del Pozzo, T. G. F. Li and C. Messenger, "Cosmological inference using gravitational wave observations alone," arXiv:1506.06590 [gr-qc].

[17] R. G. Cai, Z. Cao, Z. K. Guo, S. J. Wang and T. Yang, "The Gravitational-Wave Physics," Natl. Sci. Rev. 4, 687 (2017) doi:10.1093/nsr/nwx029 [arXiv:1703.00187 [grqc]].

[18] B. P. Abbott et al. [LIGO Scientific and Virgo Collaborations], "GW170817: Observation of Gravitational Waves from a Binary Neutron Star Inspiral," Phys. Rev. Lett. 119, no. 16, 161101 (2017) doi:10.1103/PhysRevLett.119.161101 [arXiv:1710.05832 [gr-qc]].

[19] B. P. Abbott et al. [LIGO Scientific and Virgo and 1M2H and Dark Energy Camera GW-E and DES and DLT40 and Las Cumbres Observatory and VINROUGE and MASTER Collaborations], "A gravitational-wave standard siren measurement of the Hubble constant," Nature 551, no. 7678, 85 (2017) doi:10.1038/nature24471 [arXiv:1710.05835 [astro-ph.CO]].

[20] S. Nissanke, D. E. Holz, S. A. Hughes, N. Dalal and J. L. Sievers, "Exploring short gamma-ray bursts as gravitational-wave standard sirens," Astrophys. J. 725, 496 (2010) doi:10.1088/0004-637X/725/1/496 [arXiv:0904.1017 [astro-ph.CO]].

[21] W. Zhao, C. Van Den Broeck, D. Baskaran and T. G. F. Li, "Determination of Dark Energy by the Einstein Telescope: Comparing with CMB, BAO and SNIa Observations," Phys. Rev. D 83, 023005 (2011) doi:10.1103/PhysRevD.83.023005 [arXiv:1009.0206 [astro-ph.CO]].

[22] N. Tamanini, C. Caprini, E. Barausse, A. Sesana, A. Klein and A. Petiteau, "Science with the spacebased interferometer eLISA. III: Probing the expansion 
of the Universe using gravitational wave standard sirens," JCAP 1604, no. 04, 002 (2016) doi:10.1088/14757516/2016/04/002 [arXiv:1601.07112 [astro-ph.CO]].

[23] R. G. Cai and T. Yang, "Estimating cosmological parameters by the simulated data of gravitational waves from the Einstein Telescope," Phys. Rev. D 95, no. 4, 044024 (2017) doi:10.1103/PhysRevD.95.044024 [arXiv:1608.08008 [astro-ph.CO]].

[24] R. G. Cai, N. Tamanini and T. Yang, "Reconstructing the dark sector interaction with LISA," JCAP 1705, no. 05, 031 (2017) doi:10.1088/1475-7516/2017/05/031 [arXiv:1703.07323 [astro-ph.CO]].

[25] R. G. Cai and T. Yang, "Standard sirens and dark sector with Gaussian process," EPJ Web Conf. 168, 01008 (2018) doi:10.1051/epjconf/201816801008 [arXiv:1709.00837 [astro-ph.CO]].

[26] W. Zhao and L. Wen, "Localization accuracy of compact binary coalescences detected by the third-generation gravitational-wave detectors and implication for cosmology," Phys. Rev. D 97, no. 6, 064031 (2018) doi:10.1103/PhysRevD.97.064031 [arXiv:1710.05325 [astro-ph.CO]].

[27] R. G. Cai, T. B. Liu, X. W. Liu, S. J. Wang and T. Yang, "Probing cosmic anisotropy with gravitational wave as standard siren," arXiv:1712.00952 [astro-ph.CO].

[28] S. M. Feeney, H. V. Peiris, A. R. Williamson, S. M. Nissanke, D. J. Mortlock, J. Alsing and D. Scolnic, "Prospects for resolving the Hubble constant tension with standard sirens," arXiv:1802.03404 [astro-ph.CO].

[29] L. F. Wang, X. N. Zhang, J. F. Zhang and X. Zhang, "Impacts of gravitational-wave standard siren observation of the Einstein Telescope on weighing neutrinos in cosmology," Phys. Lett. B 782, 87 (2018) doi:10.1016/j.physletb.2018.05.027 [arXiv:1802.04720 [astro-ph.CO]].

[30] Einstein gravitational wave Telescope conceptual design study, http://www.et-gw.eu/et/.

[31] F. Beutler et al., "The 6dF Galaxy Survey: Baryon Acoustic Oscillations and the Local Hubble Constant," Mon. Not. Roy. Astron. Soc. 416, 3017 (2011) doi:10.1111/j.1365-2966.2011.19250.x [arXiv:1106.3366 [astro-ph.CO]].

[32] A. J. Ross, L. Samushia, C. Howlett, W. J. Percival, A. Burden and M. Manera, "The clustering of the SDSS DR7 main Galaxy sample C I. A 4 per cent distance measure at $z=0.15$," Mon. Not. Roy. Astron. Soc. 449, no. 1, 835 (2015) doi:10.1093/mnras/stv154 [arXiv:1409.3242 [astro-ph.CO]].

[33] A. J. Cuesta et al., "The clustering of galaxies in the SDSS-III Baryon Oscillation Spectroscopic Survey: Baryon Acoustic Oscillations in the correlation function of LOWZ and CMASS galaxies in Data Release 12," Mon. Not. Roy. Astron. Soc. 457, no. 2, 1770 (2016) doi:10.1093/mnras/stw066 [arXiv:1509.06371 [astro-ph.CO]].

[34] M. Betoule et al. [SDSS Collaboration], "Improved cosmological constraints from a joint analysis of the SDSS-II and SNLS supernova samples," Astron. Astrophys. 568, A22 (2014) doi:10.1051/0004-6361/201423413 [arXiv:1401.4064 [astro-ph.CO]].

[35] A. Lewis and S. Bridle, "Cosmological parameters from CMB and other data: A Monte Carlo approach," Phys. Rev. D 66, 103511 (2002) doi:10.1103/PhysRevD.66.103511 [astro-ph/0205436]. 